\title{
Study on the Key Technology of GIS/GPS Data Pretreatment for Traffic
}

\section{Condition Detection}

\author{
Yanlong $\mathrm{Chu}^{1 \mathrm{a}}$ \\ ${ }^{1}$ Police Administration Department, Liaoning Police Academy, Liaoning, Dalian, 116036 \\ a email
}

KEYWORDS: GIS; GPS; Road Condition; Monitoring System

\begin{abstract}
With vehicles with vehicle-mounted GPS device gradually increased, GPS floating car technology has become a new type of road traffic state detection method. GPS and GIS data preprocessing is to achieve the basic link GPS floating vehicle technology and can provide more reliable data source for road traffic state detection. All of those have a direct impact on the efficiency and accuracy of the traffic state detection. Therefore, in-depth study of GPS and GIS data preprocessing key technology for GPS-based floating car road traffic status detection is much meaning and important.
\end{abstract}

\section{Introduction}

Detection of the traffic state is to achieve road traffic guidance and traffic control, improving the basic road traffic conditions; citizens can travel to the city, urban public transport scheduling reference. Meanwhile, the state of road traffic law as the basis for urban traffic data, but also provide a basis for planning and design of urban road traffic planning and development and urban construction. Floating car road traffic monitoring technology is one of the Intelligent Transportation System typical application technology. Floating vehicle generally refers to the main road in the city and with a vehicle mounted car GPS positioning device. Floating car provides real-time vehicle location, speed running state information can be used to detect and estimate the actual road traffic status information. In addition to the internal floating vehicle equipped with positioning and wireless communication devices, the operation mode and the ordinary vehicles without any distinction, without any negative impact on the traffic environment, to the monitoring center can provide on-site traffic data, while real time traffic information to achieve traffic command scheduling, improve operational efficiency of road. Floating car traffic information collects with a short construction period, less investment, wide coverage and high data accuracy, Real-time performance and other advantages of the traditional traffic information collection methods useful complement. Meanwhile, based on China's urban road traffic to improve efficiency and green development concept, and give full play to our advantage, combined with the existing urban road information detecting means used in research and in favor of floating car traffic information detection and management will be realized an important way to urban intelligent transportation.

\section{Related Technology Overview}

Geographic Information System (GIS) is a collection of modern computer science, geography, information science, management science, and surveying and mapping as one of an emerging 
discipline. It uses databases, computer graphics, multimedia and other latest technology, geographic information processing, real-time accurate capture, modify and update geospatial data and attribute information for policy-makers to provide visual support, it can be said is a GlS decision support system, with all the characteristics of information systems. But the main difference GIS with other information systems in the information stored thereon is geocoded, location and land features and attribute information relating to the position to become an important part of information retrieval. It GIS its unique geospatial features and various statistics expressive, so widely applied to various fields GIS society, such as transportation, land use, resource management, environmental monitoring side, urban planning.

GIS is an acquisition, access, edit, process, analyze, and display geographic data output system. It has seven functions: data collection, integration, entry and editing, data, data reconstruction and data conversion, query and retrieval of spatial data, spatial operation and analysis, as well as space to display and output the results of spatial data updates.

GPS is an acronym English Navigation satellite Timing and Ranging / Global Positioning system of NAVSTAR / GPS for short. Now the international community has recognized: that the global positioning system referred to as GPS. GPS consists of three separate parts: Space segment. Ground support portion; »user segment. Space segment The space segment, also known as GPS satellite constellation, satellite navigation by the 24 US Department of Defense launched composition, evenly distributed in six orbital planes, ensuring a minimum anywhere on Earth can be seen four navigation satellites. Ground floor supporting portion supporting portion also known as ground monitoring section, its role is uninterrupted observations of GPS satellites, information and calculations and forecasts by the implantation station for satellite information update. User segment user segment (GPS) receiver, GPS signals it receives to transform, amplification and processing and interpretation of the GPS satellite navigation message sent in real time to calculate the position of the measuring point, velocity and time.

\section{Map Database Design and Elevation Map}

Map pretreatment primarily to reduce the redundancy of the map data, improve the reliability of the map data, combined with the actual situation of the road on the road to a reasonable segmentation and, if necessary subject to the segmented map data for special treatment, so that follow-up work can be effectively carried out. Quality map data will directly affect the effect of map matching, and ultimately affect the result of the road traffic condition assessment of reliability. In order to obtain a GPS-based map data to meet the floating vehicle road traffic information processing requirements, first of all, the need to research, the study area and delineation of Road. Secondly, major roads within the study area-wide map-correction, namely: Construction of the road network topology, road edges node automatically generated line segment direction angle calculation, optimization of the linear line and the like. Finally, the road to the actual situation, important traffic junctions, traffic lights and bus stops location within the study area sections of trunk roads divide reasonable.

Property Data larger than the present system involves a lot of traffic infrastructure and a large number of features on the basis of information, including sections of the site, the intersection and the like. Each map layer attribute fields contain corresponding internal attribute data table file (TAB file and .DAT files), in the design attribute data, the design of the first layer of the object (entity) of (Table 1 shows attribute data table), then in accordance with the relevant attribute fields added to the layer determined the object field information. For some attribute information constantly changing, the outside of a relational database, so it needs to establish the corresponding relational 
data tables are stored. In addition, an object can correspond to the remote database several data appearances. Table 2 is a remote database attribute table.

Table 1. Attribute data table of station Layer

\begin{tabular}{|l|l|l|l|}
\hline \multicolumn{1}{|c|}{ Node No. } & Node Name & The Location No. & The Direction \\
\hline 001 & Pine Bridge & 005 & 1 \\
\hline$\cdots \cdots$ & $\cdots \cdots$ & $\cdots \cdots$ & $\cdots \cdots$ \\
\hline
\end{tabular}

Table2 Road section node information data table

\begin{tabular}{|l|l|l|l|l|}
\hline $\begin{array}{l}\text { Field } \\
\text { No. }\end{array}$ & Field name & $\begin{array}{l}\text { Data } \\
\text { catagory }\end{array}$ & Field Lenth & Note \\
\hline 1 & Node number & Int & 4 & Key Field \\
\hline 2 & Link node longitude & float & 8 & \\
\hline 3 & Link node latitude & float & 8 & \\
\hline 4 & Sections of the left line node longitude & float & 8 & \\
\hline 5 & Sections of the left line node latitude & float & 8 & \\
\hline 6 & Link on the right line node longitude & float & 8 & \\
\hline 7 & Link on the right line node latitude & float & 8 & \\
\hline$\cdots \cdots .$. & $\cdots \cdots$ & $\cdots \cdots$ & $\cdots \cdots$ & $\cdots \cdots$ \\
\hline
\end{tabular}

The basic idea of the algorithm is: the establishment of a buffer, draw a circle, draw arc method uses MapX provided strike nodes, each link shape point corresponding sections sideline node.

Step1 link layer is removed from a section of L-, see the section of the node number and shape of the point $n$, if $n>2$, turn st namely 2; if $n=2$, turn Step5

Step2: sequentially computing sections L, the first 2 to $n-1$ points (dot shape) corresponding to the edges of the road node (assuming the road width $40 \mathrm{~m}$ ). First, do sections L, buffer (buffer width of $20 \mathrm{~m}$ ); then sections $\mathrm{L}$, only the first node $\mathrm{j}$ as the center, $10 \mathrm{~m}$ radius circle, cross sections in $\mathrm{A}, \mathrm{B}$ two points, and then use MapX provided methods a, B two paintings arc ACB, point $C$ is the midpoint of the arc AcB (the point coordinates can be extracted from the MapX arc ACB), the connection c, $\mathrm{p}$ and pay the same token buffer at point $\mathrm{D}$ to point $\mathrm{E}$ method for finding .

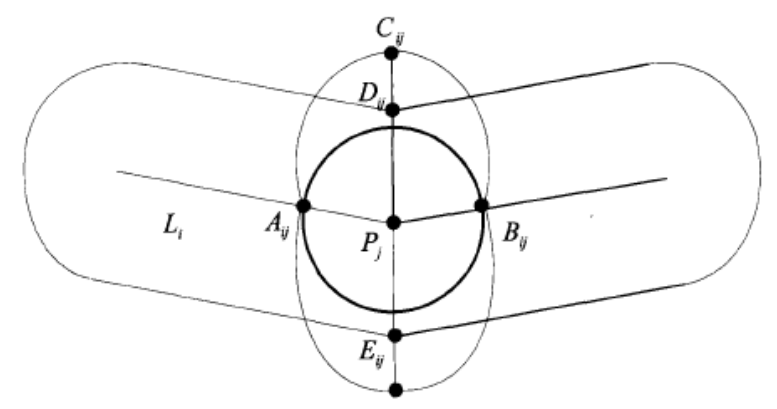

Fig.1 The situation of computing side line node of segment shape spot

Step3: in the link layer, link the object's first search link L at node p, if the object is greater than the number of sections 1, turn SteP4; otherwise, the first compute node only, road edges corresponding nodes (nodes are calculated as shown only 2 corresponds to the node only two road edges, 2 double offset relative to only two, and then the first node king, coordinate with the corresponding offset coordinates of that first node monarch, road edges corresponding node D, E formula, ( calculation method tail node empathy) turn step6: 


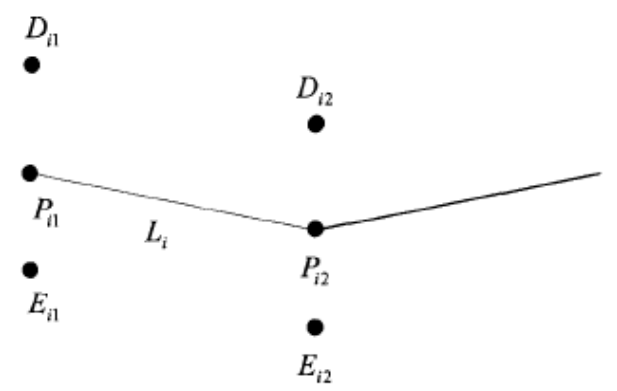

Fig.2 The situation of computing side line node of segment head and tail node Step 4: When the number of sections of the object at the first node is greater than 1 :

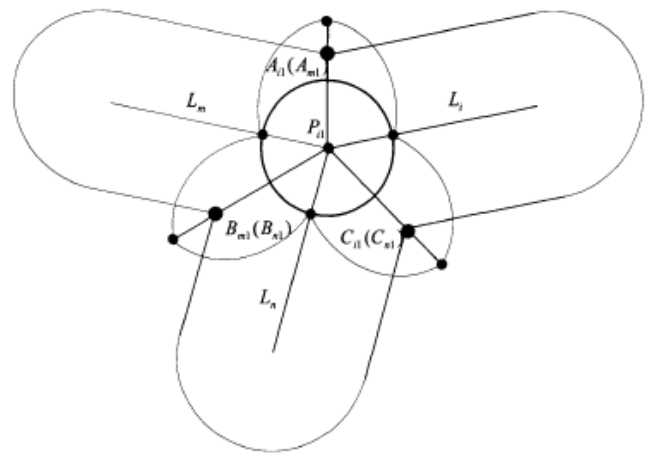

Fig.3 The situation of Computing side line node of segment head and tail node ll

Step5: When the link L- shape nodes and the number of points $n=2$, find the midpoint of these two points, and referred to as the midpoint of the first two sections of the L- node, then 2 Methods St knock calculate the midpoint of the corresponding sections of the sideline node. Turn step3.

Step6: $i=i+1$, if $i$ is greater than the total number of layers in the road, then exit; otherwise, turn step1.

Since the receiver from the GPS terminal to the GPS data does not include road, straight sections and pieces of information, it can not be directly available to the estimated average speed, we need to apply the map-matching algorithm to match GPS data to a different road, on the road and straight line segments ; since the state road traffic in both directions of different two-way street, we need to research the vehicle traveling direction determination method for classifying each section of the vehicle in both directions.

The following recent point estimate map-matching algorithm as an example to illustrate this map matching algorithm implementation process, in order to identify deficiencies and the reasons for the presence of an existing map-matching algorithm is not suitable for this project. Nearest point estimation map matching algorithm is a relatively simple map-matching algorithm, the main idea is around the GPS positioning points to a certain radius search link, then the GPS positioning point vertically projected onto the road each strip search obtained compare the pedal distance, take a minimum as the matching results. The shortest distance is a road that matched sections, GPS positioning pedal point on the road is the specific location of that point after the match. 


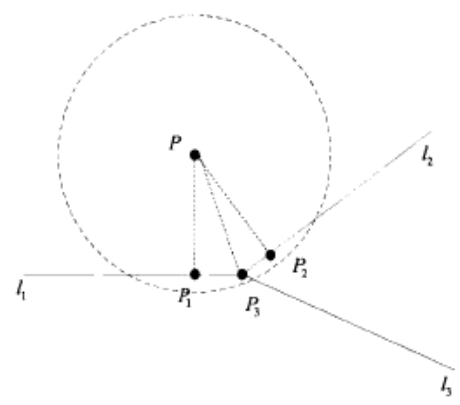

Fig.4 The nearest Point estimated map matching

Figure 4 is a schematic map of the nearest point on the estimated matching algorithm (map matching the inner layer objects to link objects). GPS positioning point $\mathrm{p}$ to the maximum error as radius circle map search, you can search to three sections 11, L2 and 13. GPS positioning point p do these three sections of the vertical projection, won three pedal points p1, p2 and p3, respectively. Because of the few pedal several reverse the extension line, so the only point positioned at the apex of a few . Comparison of three pedal distance, take a minimum as a result.

Slope k can be calculated:

$$
\mathrm{k}=\frac{p_{2 y}-p_{1 y}}{p_{2 x}-p_{1 x}}
$$

After obtaining the slope $\mathrm{k}$, calculated by the following formula,:

$$
\begin{aligned}
& p_{3 x}=\frac{k^{*}\left(p_{3 y}-p_{1 y}\right)+\left(p_{3 x}-p_{1 x}\right)}{k^{2}+1}+p_{1 x} \\
& p_{3 y}=\frac{k^{*}\left(p_{3 y}-p_{1 y}\right)+\left(p_{3 x}-p_{1 x}\right)}{k^{2}+1}+p_{1 y}
\end{aligned}
$$

From the implementation of the above algorithm, we can see that: the nearest point on the map-matching algorithm is estimated as a relatively simple, small system load algorithms, yet three steps to complete the calculation of the final match: Select a segment to determine which one is located off the road on line, calculated match point. Select a segment can be done by MapX search; determining situated on the need to calculate which of the $\mathrm{n}$ sections of a line segment of each pedal once and comparing n-1 times to determine which line segment (n nodes and shape of the section of a point number, $\mathrm{n} 1$ is a number of segments of the folded sections), if the candidate is the $\mathrm{m}$ sections, we need to calculate, compare the m (n-1) times. If you improve the accuracy of matching also require more complex algorithms.

\section{Conclusion}

In-depth analysis of Maphifo paper format for electronic maps, GPS raw data and map-matching algorithm based on the existing, against the background of the research and application requirements proposed GPS and GIS data preprocessing program. Focus on the electronic map pretreatment, GPS data exception handling and map-matching algorithm. For the core issues need to be addressed map pre-processing stage, the research process map key technologies: depending on application requirements, the map database is designed to achieve a correlation of spatial data and attribute data; Maplnfo format for electronic maps do not have the topology of the road network does not communication problem, based on the construction of road network information preprocessing urban road network topology algorithm; studied sections sideline node automatic 
generation algorithm, to meet the new map matching algorithm and dynamic traffic state GIS display requirements for road sideline node information.

\section{Acknowledgement}

Proj: General Research Project of 2015 Liaoning Province, Department of Education Science, Road Traffic Meteorological Monitoring and Warning System based on GIS.

Project NO.: L2015246.

\section{REFERENCE:}

[1] Zhou D S. An integrated traffic incident detection model [D] Austin:. University of Texas, 2000.

[2] Schrank D, Lomax T, Eisele B. The 2010 Urban Mobility Report [R]. Texas Transportation Institute, Texas, 2011.

[3] Payne H J, Helfenbein E D, Knobel, H C. Development and testing of incident detection algorithms [J]. Virginia:. 2, Research methodology and detailed results, 1976.

[4] Jacobson L N, Nihan N L, Bender J D. Detecting erroneous loop detector data in a freeway traffic management system [J]. Transportation Research Record 1287, TRB, National Research Council, Washington, D.C., 1990: 151-166. 